Revista de Psicología Vol. 32 (1), 2014 (ISSN 0254-9247)

\title{
La violencia delincuencial asociada a la salud mental en la población salvadoreña ${ }^{1}$
}

\author{
José Ricardo Gutiérrez Quintanilla ${ }^{2}$ y \\ Cecilia Beatriz Portillo García ${ }^{3}$ \\ Universidad Tecnológica de El Salvador - El Salvador
}

El presente es un estudio expos facto con un diseño retrospectivo y transeccional, que consideró una muestra probabilística de 1,143 personas (538 hombres y 605 mujeres) con un rango de edad entre 17 y 56 años o más. Los instrumentos de medida fueron la Escala de estrés y ansiedad delincuencial, y el Cuestionario de salud general. Los resultados revelaron que aproximadamente las tres cuartas partes de la muestra consideró que el contexto de violencia delincuencial afectó su salud mental. Existen diferencias entre hombres y mujeres. Las mujeres y los residentes urbanos presentaron mayor número de síntomas de estrés y ansiedad delincuencial. Se sugieren recomendaciones para estudios futuros Palabras clave: Violencia delincuencial, estrés, ansiedad, salud mental

\section{Criminal violence associated to mental health in Salvadorian people}

This is an ex post facto study with a retrospective and cross sectional design, consisting of a random sample of 1,143 participants (538 men and 605 women), age range 17-56 or more. The measures used involved the Escala de estrés y ansiedad delincuencial, and the Cuestionario de salud general. Results revealed that approximately three fourths of the simple

1 Nota del Autor: El presente artículo es un extracto del estudio La violencia social delincuencial asociada a la salud mental en los salvadoreños, realizado con el apoyo de la Universidad Tecnológica de El Salvador y la colaboración de Arely Villalta de Parada (Decana de la facultad de CC.SS), Francisco Armando Zepeda (Director del CIOPS), Edgardo Chacón Andrade (Director Escuela de Psicología) y Julio Cesar Martínez (Director Escuela de Antropología), cuyo objetivo fue determinar si existe relación entre la violencia delincuencial con la salud mental en los salvadoreños.

2 Doctor en Psicología (Ph.D), Profesor e investigador de la Vicerrectoría de investigación (VRI) de la Universidad Tecnológica de El Salvador (UTEC). Dirección postal: Calle Arce y 17 avenida norte, No. 954, Edifico José Martí, 2a y 3a. Planta, San Salvador, El Salvador, CA. Contacto: jose.gutierrez@utec.edu.sv

3 Licenciada en Psicología, pasante de investigación en la Vicerrectoría de investigación (VRI) de la Universidad Tecnológica de El Salvador (UTEC). Dirección postal: Calle Arce y 17 avenida norte, No. 954, Edifico José Martí, 2a y 3a . Planta, San Salvador, El Salvador, CA. Contacto: cciliaportillo@gmail.com 
considered that the criminal violence context affected their mental health. Differences between men and women were established. Women and urban residents exhibited a greater number of stress symptoms and criminal anxiety. Recommendations for future studies are suggested.

Keywords: criminal violence, stress, anxiety, mental health 
En los últimos años, la tasa de homicidios registrados en El Salvador lo convirtió en uno de los países más violentos del mundo. Sin embargo, desde marzo de 2012, el número de homicidios ha disminuido considerablemente como resultado de una tregua entre pandillas. No obstante, las cifras de los muertos por actos violentos y delincuencia reflejan la dimensión extrema del problema. Diariamente se comenten diferentes actos que implican violencia, tales como: abusos físicos, psicológicos y sexuales, los cuales perjudican la salud y el bienestar de las víctimas (OMS, 2002); incluyendo a las víctimas indirectas que conviven el dolor de sus familiares, amigos y vecinos, y a la población general que vive con temor a ser víctima directa de la delincuencia (Falkenburger \& Thale, 2008).

Al mismo tiempo, dentro de las repercusiones de la violencia, se hallan los altos costos que supone para el Estado, la atención sanitaria, judicial, absentismo laboral y pérdida de la productividad del país (OMS, 2002). Por lo tanto, no basta con sólo conocer el número de homicidios para medir, explicar y reducir el problema de la violencia y delincuencia; es importante enfatizar las repercusiones que impone a nivel individual, a nivel social y económico del país (Morales, Muñoz, Santillán, Arenas \& Ponce, 2007); así como conocer los factores que pueden estar involucrados o incidiendo en el incremento de la violencia delincuencial, como lo son: el incremento de las armas de fuego, la desigualdad social, el desempleo y el acostumbramiento de las personas a la violencia (Briceño, 2007; OMS, 2002).

La violencia es un fenómeno que marca la historia social, familiar y personal (Saavedra, 2004). En El Salvador, la violencia social delincuencial es uno de los temas de mayor preocupación, debido a que una proporción importante de la población está siendo víctima de una cantidad de delitos como: extorsiones, amenazas, robos, hurtos, asaltos, lesiones y control territorial de las pandillas o maras. A este contexto 
de violencia se suma el influjo psicológico que ejercen los medios masivos de comunicación social, al presentar constantemente las noticias de homicidios y describir con detalle la crueldad de los actos violentos (como el desmembramiento de las víctimas), influyendo en muchas ocasiones en el miedo de las comunidades con presencia de pandillas (Falkenburger \& Thale, 2008); además de producir en la población diferentes sentimientos y emociones como son: el miedo, la inseguridad, la ansiedad, la angustia, la inestabilidad física y psíquica, y los cambios de conducta y de estilos de vida (no salir de noche, llegar temprano a casa, cerrar temprano las puertas de la casa, etc.).

Por otra parte, el problema de la violencia social delincuencial también presiona al gobierno a buscar respuestas inmediatas para afrontar el problema, empleando el accionar policial con capturas masivas y el encarcelamiento de jóvenes delincuentes (o no), que en la mayoría de casos quedan en libertad por falta de pruebas, pero que tienen como objetivo ofrecer a los ciudadanos una sensación inmediata de mayor seguridad (Falkenburger \& Thale, 2008). El contexto social delincuencial que experimenta una proporción importante de salvadoreños, probablemente está afectando la salud mental, la calidad de vida, la convivencia ciudadana y el derecho al esparcimiento saludable de la población. Es desde esta realidad no investigada que surgió la necesidad, la motivación e importancia de analizar el estado de salud mental de los salvadoreños asociado al contexto de violencia social delincuencial.

La Universidad Tecnológica de El Salvador (UTEC), con la pretensión de demostrar esta conexión, desarrolló un estudio a escala nacional que fue presentado en el año 2012, titulado: La violencia social delincuencial asociada a la salud mental en los salvadoreños. El estudio fue motivado por dos razones básicas: la primera es que no existen estudios empíricos en El Salvador que expliquen la relación de un contexto de violencia social delincuencial con la salud mental de la población general y de las víctimas directas o indirectas de la violencia delincuencial. La segunda es debido a que en estudios de opinión se han encontrado índices que superan el 30\% de víctimas de la violencia delincuencial, y tasas de homicidios registrados por la Policía Nacional Civil (PNC) que 
superan los 65 homicidios por cada 100 mil habitantes (PNC, 2011). Asimismo, el país no cuenta con un sistema integrado de estadísticas e información sobre el fenómeno de la violencia.

En la historia de la humanidad, el siglo XX fue uno de los periodos más violentos (OMS, 2002). En los últimos años, la prevención de la violencia, ha sido un tema prioritario para los sistemas de salud debido a la repercusión de este fenómeno en la salud de las víctimas y a los costos que impone a las instituciones sanitarias. Por lo tanto, el sector sanitario tiene un papel central que desempeñar en la disminución de la violencia (OMS, 2002), de tal manera que el problema debe ser abordado desde el enfoque de la salud pública (OMS, 2003). Por ello, la violencia fue declarada como un problema de salud pública, lo que implica un tratamiento desde diversas perspectivas para reducir sus niveles de incidencia.

En la década del ochenta, la tasa de homicidios se duplicó en la mayoría de los países (Briseño, 2007). En el año 2000 alrededor de 1.6 millones de personas perdieron la vida en todo el mundo a causa de actos violentos (OMS, 2002). Aproximadamente, la mitad de estas muertes se debieron a suicidios (en su mayoría hombres), casi una tercera parte a homicidios y una quinta parte a conflictos armados (OMS, 2002). En el informe emitido por la Declaración de Ginebra sobre violencia armada y desarrollo titulada Carga Global de la Violencia Armada (La Declaración de Ginebra, 2011) se señala que a nivel mundial cada año mueren más de 526,000 personas a consecuencia de la violencia letal. También se registran cada ańo 55,000 muertes directas resultantes de conflictos y 396.000 víctimas de homicidios intencionales. Entre los años 2000 y 2009, los datos por país señalan que a mayor diferencia de ingresos económicos, mayores tasas de homicidios. En los países con grandes desigualdades económicas, las tasas de homicidios relacionados con robos y asaltos tienden a ser mayores. En América Central y del Sur, el porcentaje de homicidios relacionados con las pandillas o el crimen organizado es considerablemente más alto que en Asia y Europa (La Declaración de Ginebra, 2011).

Según el Banco Mundial (BM, 2011), las tres causas principales de la violencia en la región Centroamericana son: el tráfico de drogas, 
la disponibilidad de armas de fuego, la violencia juvenil o las maras. Los hombres de entre 15 y 34 años de edad constituyen la abrumadora mayoría de las víctimas de homicidio, que también forman parte de las pandillas juveniles. En un informe de la Agencia de Estados Unidos para el Desarrollo Internacional (United States Agency for International Development, USAID), en el que se evalúa la magnitud del fenómeno de la violencia en cinco países de la región (México, Guatemala, El Salvador, Honduras y Nicaragua), se estimó que el número de miembros de maras en dichos países podría alcanzar los 300 mil, y que su número está ascendiendo (USAID, 2006). En dicho informe, El Salvador registra una tasa de 180 pandilleros por cada 100 mil personas. Las maras o pandillas, sin duda alguna, son responsables en gran parte del contexto de violencia en El Salvador, Guatemala y Honduras. Entre los factores que han contribuido de forma directa en la violencia juvenil en El Salvador se encuentran: las condiciones de subdesarrollo, la migración, la posguerra, la violencia doméstica, unas instituciones débiles, la inestabilidad económica y la pobreza extrema. Cabe destacar que los jóvenes que conforman la maras o pandillas, en su mayoría provienen de estratos sociales bajos en condiciones de marginalidad, y habitan principalmente en zonas urbanas y suburbana (Cabezas, Gilsanz \& Sampayo, 2008; Falkenburger \& Thale, 2008).

La Declaración de Ginebra (2011) reveló que El Salvador fue el país más afectado por la violencia letal entre los años 2004 y 2009, seguido por Irak y Jamaica, además señaló que en países como El Salvador y Guatemala una cantidad considerable de mujeres y niñas son víctimas de asesinatos. Los países que registran tasas más altas de feminicidios también registran tasas altas de homicidios. Los países con altos porcentajes de homicidios cometidos con armas de fuego, también registran tasas globales más altas de muertes violentas. Las armas de fuego se han convertido en las armas preferidas por el crimen organizado y las pandillas, siendo el uso de armas de fuego más frecuente en los homicidios que en los feminicidios (La Declaración de Ginebra, 2011).

En El Salvador, según información de la base de datos conjunta del Instituto de Medicina Legal (IML), la Fiscalía General de la República 
(FGR) y la Policía Nacional Civil (PNC), en el año 2006 se registraron 3,928 homicidios a escala nacional (PNC, 2011), lo cual implicaría una tasa de casi 68 homicidios por cada 100 mil habitantes, esto es 6.8 veces la tasa considerada como epidémica por la Organización Panamericana de la Salud (OPS). De acuerdo con la OPS, un índice «normal» de criminalidad es el que se halla entre 0 y 5 homicidios por cada 100 mil habitantes por ańo. Cuando el índice de homicidios excede a 10, una sociedad se enfrenta a un cuadro de criminalidad "epidémica". En 2007, el número de homicidios había disminuido a 3,497, con lo cual la tasa correspondiente también se habría reducido a un poco más de 61 homicidios por cada 100 mil habitantes. En el año 2010 el número de homicidios fue de 3,987, con una tasa de 69.9 homicidios por cada 100 mil habitantes, observándose un incremento con relación al 2006 y al 2007. Cabe señalar que en el año 2009, estas cifras se dispararon con una incidencia de 4.382 homicidios y una tasa de 76.9 homicidios por cada 100 mil habitantes.

En América Latina las estadísticas oficiales del número de homicidios no son fieles, debido a que muchas muertes se disfrazan de accidentes o se atribuyen a enfermedades y causas naturales (OMS, 2002). Este contexto de violencia ha creado un sentimiento de inseguridad muy alto en las distintas zonas de la ciudad, alterando los estilos de vida de los ciudadanos con el fin adaptarse a las condiciones de inseguridad en las que están inmersos (Briceño, 2007), por ejemplo el abandono de muchas zonas (colonias, barrios) por temor a ser víctimas de la delincuencia, las restricciones de acceso a colonias, los condominios vigilados, el pagar vigilancia privada o el no frecuentar lugares identificados como peligrosos (calles o ciudades). También existen personas que compran armas o sistemas de vigilancia remota, se organizan en comités de vigilancia dentro de las colonias, como respuestas a un ambiente social amenazante y de alto riesgo delictivo (Huhn, Oettler \& Peetz, 2007).

El Instituto Universitario de Opinión Pública (IUDOP, 2007) de la Universidad Centroamericana (UCA), señalaba que «la situación de crimen e inseguridad sigue siendo motivo de enorme preocupación 
ciudadana», y es el resultado de la situación delincuencial del país. Para finales del 2007, más de la mitad de las personas encuestadas (52.4\%) expresaron que la violencia aumentó respecto al año anterior, en este estudio el $19.4 \%$ de las personas declaró haber sido víctima de un hecho delincuencial de forma directa. Según la encuesta del Centro de Investigación de la Opinión Pública Salvadoreña (CIOPS, 2011), un $30.9 \%$ de la muestra fue víctima directa de la delincuencia pandilleril en la zona de residencia. Entre los tipos de delitos cometidos más frecuentemente, se encontró que $23.5 \%$ fueron hurtos y robos, y $18.8 \%$ extorsiones. Asimismo, 36.1\% manifestó haber sido víctima de robo en la calle de la ciudad. Por otra parte, se encontró que $47.6 \%$ de la población salvadoreña manifestó que la delincuencia se había incrementado en el país. En el mismo estudio, se expresó que $82.3 \%$ de la población tiene la percepción de que la sociedad salvadoreña estaba dominada por la delincuencia.

En un estudio realizado a escala nacional (Gutiérrez, 2011), se encontró que la ansiedad y la depresión son las alteraciones mentales más prevalentes en la población salvadoreña. Más de la mitad de la muestra presentó síntomas de ansiedad e insomnio, hallando mayor incidencia de síntomas en las mujeres y en los habitantes de las zonas rurales. El informe revela la existencia de $35.1 \%$ de casos de psicopatologías afectivas en la población general salvadoreña, disminuyendo la presencia de sintomatología patológica a la medida que se incrementa el nivel educativo de las personas. En los años 2001, 2002, 2005 el trastorno de ansiedad fue la primera causa de consulta de los trastornos mentales y del comportamiento en el sistema nacional de salud (Ministerio de Salud, MINSAL, 2003, 2010, 2012). Asimismo, para los años 2006-2012, la ansiedad y la depresión aparecen en primer y segundo lugar respectivamente como las principales causas de consulta de los trastornos mentales (MINSAL, 2012). Estos datos indican la prevalencia de problemas de salud mental en la población salvadoreña, aunque estos problemas de salud mental no se pudieron vincular con la violencia delincuencial. En el presente artículo tiene como interés estudiar el estrés y la ansiedad delincuencial, como una consecuencia psicológica 
y emocional del contexto de violencia delincuencial, como indicadores de problemas de salud mental en la población que fue víctima directa o indirecta de la violencia delincuencial. Por lo tanto, es relevante realizar una aproximación teórica al fenómeno, comenzando por definir que es la salud mental.

La OMS define la salud mental como un estado de bienestar en donde la persona es consciente de sus propias capacidades, puede afrontar las tensiones normales de la vida, trabajar productivamente y es capaz de hacer una contribución a su comunidad (OMS, 2011). En este sentido, la salud mental es la base para el bienestar y funcionamiento efectivo de una persona y una sociedad (Moll, 2013). Mientras que, por otro lado, la violencia hace alusión al uso de alguna forma de fuerza (sea verbal, física, política, económica.) para producir daño (Saavedra, 2004). Para este estudio, la violencia social delincuencial fue definida como la exposición constante a eventos traumáticos y estresantes, generados por la delincuencia que, con el paso del tiempo, afectan la salud mental, y la estabilidad emocional y física de quienes conviven en una comunidad. Es un contexto comunitario caracterizado por la frecuente incidencia de diferentes tipos de delitos como: homicidios, lesiones, extorsiones, robos, asaltos y secuestros, cometidos por grupos delincuenciales (las pandillas juveniles, los narcotraficantes y grupos de sicarios). Es importante recalcar que, en este estudio, el término de violencia delincuencial se utilizará como sinónimo de violencia social delincuencial, que es un contexto de violencia delincuencial que tiene el potencial de desencadenar en las personas inestabilidad emocional, estrés y ansiedad.

Tanto las víctimas directas como las víctimas indirectas presentan un mayor riesgo de sufrir problemas psicológicos tales como ansiedad, depresión y trastorno por estrés postraumático que pueden manifestarse en formas de comportamientos alterados y antisociales tales como: abuso del alcohol, comportamiento suicida, embarazos no deseados, disfunciones sexuales y enfermedades de transmisión sexual, y conflictos familiares (OMS, 2002). De la misma forma, las repercusiones de la violencia y delincuencia sobre la salud incluyen las discapacidades 
permanentes como lesiones medulares, cerebrales, o mutilaciones (OMS, 2002). Cabe señalar que la violencia y la delincuencia a menudo se asocia a diversos problemas psicológicos y comportamentales, tanto del agresor como de las victimas (OMS, 2002). Saavedra (2004) menciona que las conductas violentas y delincuenciales tienen una relación fuerte con los problemas de salud mental, dentro de los que prevalecen patologías como los trastornos de personalidad severos, los cuadros cerebrales orgánicos (retardo mental), la psicosis, así como los problemas relacionados al alcohol y las drogas.

En la actualidad no existe un solo modelo teórico que explique las raíces de la violencia y la delincuencia (OMS, 2002), ya que tanto los factores biológicos, psicológicos y sociales se conjugan para desencadenar la conducta violenta y delictiva, afectando la salud mental. Estudios de laboratorio con animales han concluido que las tensiones sociales, la violencia y la agresividad, modifican la estructura cerebral, además de variar los neurotransmisores producir cambios hormonales (Punset, 2006). Entre los factores biológicos que intervienen en la violencia y en la conducta delictiva se pueden mencionar: (a) un córtex prefrontal (la zona del cerebro que inhibe la agresividad) que no funciona con normalidad o que presenta dificultades estructurales, lo cual puede suponer una predisposición a la conducta delictiva y de violencia; (b) una disfunción en el hipocampo podría predisponer a repetir reacciones violentas; (c) una anomalía en el funcionamiento de la amígdala cerebral (Calzada, 2007). Asimismo, se ha descubierto que cuando hay más serotonina en el cerebro las personas son menos agresivas (Punset, 2006).

Entre los factores psicológicos, la violencia está íntimamente relacionada con el modo en que se construyen los esquemas mentales sobre el mundo, la vida y las relaciones durante la infancia. Un nińo(a) que ha sufrido experiencias de maltrato, con el tiempo tiende a desarrollar esquemas sobre el mundo, la vida y sobre las relaciones, en muchas ocasiones, de un modo violento, donde únicamente hay dos opciones: ser agresor o ser víctima (Punset, 2006). Otro factor que puede influir en la conducta agresiva son los niveles intelectuales. Un cociente inte- 
lectual bajo es un indicador cognitivo del comportamiento criminal (Punset, 2006). De igual forma, Reyes (2007) en su estudio considera que tener una enfermedad mental es un hecho que influye en las reacciones violentas. Cabe señalar que existe una correlación entre el maltrato infantil, un bajo coeficiente intelectual y los comportamientos violentos (Punset, 2006).

Dentro de los factores sociales que intervienen en la conducta violenta se encuentran: el trato de los padres hacia los hijos(as), el castigo físico como modo de educación que "corrige" (Morelato, 2011) y los estímulos sociales recibidos en la niñez. Un entorno deficiente en estímulos sociales, la experiencia de maltrato y la naturalización de la violencia lleva a una estructura y funcionamiento cerebral deficiente (Punset, 2006), además de la tendencia a aprender esquemas mentales negativos o violentos y subjetivos sobre el mundo, las relaciones sociales y el bienestar psicológico (Posada \& Parales, 2012). Los niños que están más propensos a la violencia son los que experimentan u observan un comportamiento violento en el hogar (Falkenburger \& Thale, 2008). El crecer en entornos caracterizados por la violencia influye en el desarrollo y funcionamiento psicológico (Posada \& Parales, 2012). De igual forma, en situaciones donde el sistema legal de un país es incapaz de castigar o controlar el delito puede darse la tendencia a utilizar los medios agresivos como métodos para restablecer la justicia (Posada \& Parales, 2012).

Huhn et al. (2007) argumentan que los fenómenos de la violencia en sí no son los que tienen más influencia en las opiniones, en la sensación de inseguridad o en la implementación de políticas de represión en una sociedad; sino más bien los discursos y prácticas sociales. El miedo hacia algún tipo de delito en la población puede ser excesivamente grande aunque se cometa pocas veces este crimen. Es importante señalar que en una comunidad en donde con frecuencia suceden diferentes tipos de delitos (homicidios, extorsiones, robos, y secuestros) o hechos de violencia, dentro de las primeras reacciones de las personas ante dichas situaciones se encuentran los estados de estrés y de ansiedad, con las implicaciones fisiológicas y psicológicas que ello conlleva, 
que con el transcurso del tiempo podrían convertirse en cuadros de estrés post traumático o de ansiedad generalizado, lo cual tiene fuertes repercusiones para la vida y la salud mental de quien lo sufre.

El estrés es el resultado de una situación que es evaluada por quien la sufre como amenazante y que pone en peligro su bienestar (Lazarus \& Folkman, 1986). Si resulta de una situación de violencia delincuencial se hablará de "estrés delincuencial", en donde los niveles altos pueden implicar efectos perjudiciales en la salud física y mental. El Centro de Estudios sobre Estrés Humano (Centre for Studies on Human Stress) menciona que un factor de estrés es todo aquello que provoca la liberación de la hormona del estrés: el cortisol. Que puede ser provocada por factores psicológicos como: situaciones, personas, comentarios, es decir, cualquier evento que se interprete como amenazante o negativo (CSHS, 2010).

Diversos estudios realizados han demostrado que los altos niveles de estrés pueden afectar la capacidad de aprendizaje y la memoria. En una entrevista realizada por Punset (2004) a Robert Sapolsky, se menciona que desplazar agresión en otro es una respuesta para reducir el estrés, lo que implica hacer que alguien se estrese y se sienta mal. Dicha respuesta es típica tanto en animales (primates) como en humanos. De igual forma, un ambiente violento puede afectar el sistema límbico, debido a que la amígdala puede crecer menos por el estrés que suponen los maltratos. A su vez, un estudio de Slavich y Taylor (2010) determinó recientemente que la exposición a grandes cantidades de estrés podría cambiar la forma en que responde el sistema inmune a las amenazas exteriores. Estos cambios pueden abrir el camino a muchas enfermedades o infecciones y podrían desencadenar una variedad de alteraciones mentales como: ansiedad generalizada (fobias), insomnio, depresión, alteraciones psicosomáticas, y en algunos casos, trastornos de personalidad paranoide y estrés postraumático, entre otros. Es decir, si el sentimiento de carácter negativo aparece de forma constante en el sujeto, y no es tratado adecuadamente, puede conducir a un bajo rendimiento en la vida cotidiana, debilitando la salud (Olga \& Terry, 1997).

Los autores Hudd et al. (2000), encontraron que los estudiantes con niveles más altos de estrés mostraban peores hábitos de salud, 
autoestima más baja y mala percepción de su estado de salud. Del mismo modo, se ha encontrado en algunos estudios que los habitantes de la ciudad, a diferencia de otras personas, tienen más probabilidades de sufrir más estrés y enfermedades mentales (Lederbogen et al., 2011).

De la misma manera, un alto nivel de hormonas de estrés (cortisol), como se ha mencionado, pueden afectar el aprendizaje y la memoria, haciendo que una persona, frente a eventos o situaciones traumáticas, aumente su capacidad para mantener el recuerdo vivo del acontecimiento traumático, con el objetivo de que en el futuro el individuo actué de tal forma que evite encuentros similares (CSHS, 2010), como mecanismo de sobrevivencia. Esto se relaciona con el estrés postraumático (TEPT) que es una reacción patológica caracterizada por ansiedad, que ocurre posteriormente a la exposición a un evento traumático. Entre los síntomas que caracterizan este estado se tienen: el recuerdo insistente del hecho traumatizante, pesadillas constantes o pensamientos recurrentes entorno al el hecho, ilusiones de repetición del evento, evitación de pensamientos, personas o lugares que recuerden el hecho (Cervantes, Salgado de-Zinder \& Padilla, 1989), estado constante de alerta o temor, incapacidad para recordar hechos (memoria expresiva), y síntomas disociativos (escaparse de la situación, despersonalización).

En un estudio realizado por Mora et al. (2005) en México se encontró que el $68 \%$ de la población ha estado expuesta al menos a un suceso estresante en su vida; que la violación, acoso y abuso sexual son más frecuentes en mujeres, mientras que los accidentes y robos eran más frecuentes en los hombres. Además, se halló que el $2.3 \%$ de las mujeres y $.49 \%$ de los hombres presentaron un trastorno de estrés postraumático y que los sucesos con mayor manifestación de trastornos por estrés postraumático son la violación, el acoso, el secuestro y el abuso sexual.

Echeburúa, Corral, Amor, Sarasua, y Zubizarreta (1997) en su estudio realizado en una muestra de 164 víctimas de maltrato doméstico (físico y psicológico) en tratamiento, encontraron la existencia de repercusiones importantes en el ámbito de la ansiedad, especialmente, en el trastorno de estrés postraumático. Por lo tanto es importante seña- 
lar que el estrés agudo puede ser útil para ayudar a la mente y cuerpo hacer frente a diversas situaciones; sin embargo, cuando la respuesta del estrés se activa con demasiada frecuencia, es donde surgen los problemas. Muchos científicos consideran que nuestro sistema de respuesta al estrés no fue diseñado para activarse constantemente, no obstante, este se activa en reiteradas ocasiones con el simple hecho de anticiparse a factores estresantes, algunos sin que en verdad lleguen a llevarse a cabo. El estrés crónico se ha relacionado con la presión arterial alta, enfermedades cardiacas, colesterol alto, diabetes tipo II, agotamiento, la depresión y aumento de ansiedad. (CSHS, 2010).

La ansiedad es una reacción emocional ante la percepción de un peligro o amenaza, manifestándose mediante un conjunto de respuestas agrupadas en tres sistemas: cognitivo o subjetivo, fisiológico o corporal y motor, pudiendo actuar con cierta independencia (Miguel-Tobal, 1996). Para la ansiedad que resulta de la violencia social delincuencial se manejará el término de ansiedad delincuencial, efecto que pueden sufrir tanto las víctimas directas como indirectas de la violencia delincuencial.

La respuesta de ansiedad puede ser excitada, tanto por estímulos o situaciones externas, como por estímulos internos al sujeto, tales como pensamientos, ideas, imágenes, etc., que son percibidos por los individuos como peligrosos y amenazantes. Las manifestaciones cognitivas pueden implicar preocupación por lo desconocido o temor de perder el control sobre las funciones corporales. Las manifestaciones conductuales implican la evitación de situaciones inductoras de ansiedad; mientras que los cambios somáticos incluyen la respiración entrecortada, sequedad de boca, manos y pies fríos, micción frecuente, mareos, palpitaciones cardíacas, elevación de la presión sanguínea, aumento de transpiración, tensión muscular e indigestión (Sue, 1996). Los autores coinciden en señalar que la ansiedad afecta de modo directo la salud mental de las personas que la sufren. Se han encontrado relaciones entre la ansiedad y los estilos de vida que promocionan la salud (Johnson, 2002).

Kessler et al. (2003) mencionan que entre los factores asociados con la ansiedad están: ser mujer, dedicarse a las labores del hogar, estar 
jubilado, ser soltero, separado, divorciado o viudo, tener bajo nivel educacional y tener bajos ingresos económicos. Igualmente, la ansiedad se ha revelado asociada al riesgo de desarrollar ciertas patologías, como, por ejemplo, la hipertensión en las mujeres (Adler \& Matthews, 1994), o como factor de riesgo de la sobre mortalidad cardiovascular (Hansen, 2003). Como resultado de la revisión de algunos modelos teóricos, estadísticas y de estudios empíricos, es importante considerar el impacto de la violencia social delincuencial en la salud y, en especial, en la salud mental de la población salvadoreña.

El presente artículo tiene como objetivo general determinar la relación entre la violencia social delincuencial y la salud mental en la población salvadoreńa. Ante los potenciales efectos psicológicos y emocionales de la violencia social delincuencial en la población salvadoreña, en esta investigación se tienen los objetivos específicos siguientes: (a) determinar la prevalencia de violencia social delincuencial y los problemas de salud mental en los salvadoreños; (b) determinar si existen correlaciones significativas entre estrés delincuencial, ansiedad delincuencial, bienestar psicológico (salud mental) y el género de la población; (c) establecer si existen diferencias estadísticamente significativas en estrés delincuencial, ansiedad delincuencial y salud mental, en función del género y si fue víctima de violencia delincuencial; (d) aplicar un modelo de regresión logística, para explicar en un porcentaje importante la ansiedad delincuencial que experimenta la población salvadoreńa víctima de la violencia delincuencial, considerando como variables predictoras (VI): el estrés delincuencial, el género, la violencia sociocultural, los problemas de salud mental, el nivel educativo, la edad, el estado familiar; y como variable criterio (VD) la ansiedad delincuencial.

Finalmente, cabe señalar que este estudio es un extracto de un informe mayor, en donde se analiza la violencia social delincuencial asociada al estrés y la ansiedad delincuencial, como indicadores de problemas de salud mental y que son el resultado de vivir un contexto de violencia social delincuencial. 


\section{Método}

El presente estudio es de tipo expos facto (Montero \& León, 2007), con un diseño retrospectivo y transeccional.

\section{Participantes}

La técnica utilizada en la recolección de la información fue la encuesta. La muestra es probabilística representativa de las ciudades más importantes del país. Para ello, se adoptó una estimación de error del 3\% y un nivel de confianza del 95\%, obteniéndose una muestra de 1.143 personas a escala nacional. En el proceso de muestreo se consideró una distribución proporcional al número de habitantes de cada departamento. En el estudio fueron encuestados 538 hombres y 605 mujeres, constituyendo el $47.1 \%$ (hombre) y $52.9 \%$ (mujeres) de la muestra respectivamente. En cuanto a la edad, se consideraron los siguientes rangos de edad: (a) 17-25 años (22.9\%), (b) 26-35 años (26.8\%), (c) 36-45 ańos (19.6\%), (d) 46- 55 años (14.5\%) y (e) 56 años o más (15.5\%). Cabe señalar que 8 participantes no indicaron su edad. A nivel de estado civil, $35.9 \%$ de los participantes eran solteros, $38 \%$ casados, $18.9 \%$ tenían una unión libre, $2.7 \%$ eran divorciados, $3.8 \%$ viudos y el $.7 \%$ no respondió. A nivel de escolaridad, el 10.8\% reportó contar con escolaridad hasta el 3er. Grado, el 16.8\% hasta 6to. Grado, el 21.1\% hasta 9no. Grado, el 30.4\% hasta el bachillerato, el $2.4 \%$ hasta educación técnica, el $8.8 \%$ hasta educación universitaria, el $8.0 \%$ reportó analfabetismo y el $1.7 \%$ no indicó su nivel de escolaridad.

Con relación a la variable lugar de residencia, 807 (70.6\%) habitaban en zonas urbanas y 336 (29.4\%) en zonas rurales. A nivel laboral, el $64 \%$ indicó que trabajaba, mientras que el 34.9\% indicó no trabajar y el resto no respondió a esta pregunta. De los participantes que indicaron estar trabajando, el 306 trabaja en el sector público y el 426 en el sector privado. 


\section{Medidas e instrumentos de medición}

La variable víctima de violencia delincuencial, fue operacionalizada como aquella persona que ha experimentado o fue víctima directa o indirecta de la violencia delincuencial, de delitos como robos, asaltos, extorsiones, lesiones, homicidios y control territorial de colonias y barrios; todos ellos eventos que tienen el potencial de perjudicar el bienestar psicológico, físico y social de las personas. Con el fin de recoger información respecto a esta variable, se elaboró un cuestionario para efectos del presente estudio, en el que se consideró un grupo de ítems relacionados con la violencia sociocultural, el consumo de alcohol y drogas, así como con la violencia delincuencial que experimenta la población salvadoreña.

El estrés delincuencial, se operacionalizó como el estrés provocado en las víctimas directas o indirectas por la exposición constante a la violencia delincuencial de muchos salvadoreños. Y la variable ansiedad delincuencial, fue definida como el conjunto de síntomas ansiosos generados en las víctimas directas o indirectas de la violencia delincuencial; salud mental, la cual consiste en un estado de bienestar psicológico y emocional que permite tener una vida saludable y armoniosa. Con el fin de evaluar el estrés y la ansiedad delincuencial, se utilizó la Escala de estrés y ansiedad delincuencial (ESAD) (Gutiérrez-Quintanilla, 2012).

La ESAD se basa en el modelo transaccional de Lazarus y Folkman (1986), quienes plantean que el estrés es el resultado de una relación entre el individuo y su entorno, que es evaluado por la persona como amenazante y que pone en peligro su bienestar. El Análisis factorial exploratorio utilizando el análisis Kaiser-Meyer-Olkin $(\mathrm{KMO}=.86)$ y la prueba de esferidad de Bartlett $\left(X_{(78)}^{2}=5235 ; p=0.001\right)$ indicó la adecuación de los datos para este tipo de análisis. Siguiendo el procedimiento de dos factores con rotación Varimax resultó en una solución de dos factores explican el $49.99 \%$ de la varianza total explicada, presentando todos los ítems valores de saturación superiores a .40 . El primer factor explicado fue estrés delincuencial con seis ítems $(1,2,3,4,5,6)$, obteniendo un $\alpha$ de Cronbach de .77. El segundo factor fue ansiedad delincuencial con siete ítems $(7,8,9,10,11,12,13)$, obteniendo un $\alpha$ de Cronbach de 0.82 . 
El tamizaje de salud mental de la población fue realizado con el Cuestionario de salud general, GHQ-12 (Golbert, 1970), que es una medida de detección (screening) de salud mental. Esta medida evalúa dos factores: (a) el primero es la salud mental o bienestar psicológico y (b) el segundo es el funcionamiento social. El análisis psicométrico demostró que la prueba posee propiedades adecuadas de fiabilidad y validez en El Salvador. La fiabilidad mediante la consistencia interna $\alpha$ de Cronbach fue superior a .77 en ambas dimensiones.

\section{Procedimiento}

Primero, se seleccionó al equipo evaluador, consistente en estudiantes de psicología del tercer año a quienes se capacitó en el manejo de las pruebas, las generalidades del proyecto y la organización del trabajo de campo. Luego se coordinó con el director ejecutivo del Centro de Investigación de la Opinión Pública Salvadoreña (CIOPS), con la intención de revisar los instrumentos, la selección de la muestra a escala nacional y la planificación del trabajo de campo. Se organizó el recojo de información según cinco rutas por departamentos del país, a las que se asignó equipos de evaluadores bajo la supervisión directa de un profesional responsable. Luego de recogida la data, se procesó la información con el paquete estadístico SPSS, v.19, para Windows.

\section{Resultados}

Los resultados del presente artículo revelan que a nivel de violencia social delincuencial, 961 participantes, equivalentes al 84.8\% de la muestra, consideran que el ambiente de violencia social delincuencial frecuentemente les produce nerviosismo e inseguridad. A su vez, al preguntar a los participantes si la situación de violencia delincuencial que viven en el país les afectaba el sueño, 751 (65.9\%) refieren que sí les afectaba frecuentemente. En cuanto a si el contexto de violencia social delincuencial afectaba su estabilidad emocional y mental, 807 (70.7\%) consideraron que sí les afectaba como se muestra en la Tabla 1. 


\section{Tabla 1}

Frecuencias y porcentajes de la muestra que frecuentemente presentan estrés y ansiedad delincuencial, como resultado de la violencia social delincuencial, comparados por sexo

\begin{tabular}{|c|c|c|c|c|}
\hline \multirow{2}{*}{ Ítems } & \multicolumn{4}{|c|}{$\begin{array}{c}\text { Frecuentemente } \\
\text { Siempre }\end{array}$} \\
\hline & $\begin{array}{l}\text { Total } \\
(\%)\end{array}$ & $\begin{array}{c}\text { Hombres } \\
(\%)\end{array}$ & $\begin{array}{c}\text { Mujeres } \\
(\%)\end{array}$ & $X^{2}$ \\
\hline $\begin{array}{l}1 \text { ¿En el último ańo, algún } \\
\text { pariente suyo fue víctima de } \\
\text { la delincuencia (Ej.: robo, } \\
\text { hurto, extorsión)? }\end{array}$ & $498(43.8)$ & $233(46.8)$ & $265(53.2)$ & 0.017 \\
\hline $\begin{array}{l}2 \text { ¿En el último año, algún } \\
\text { amigo o conocido suyo fue } \\
\text { víctima de la delincuencia (Ej.: } \\
\text { robo, hurto, extorsión)? }\end{array}$ & $656(57.5)$ & $324(49.4)$ & $332(50.6)$ & $3.756 \dagger$ \\
\hline $\begin{array}{l}3 \text { ¿En el último año, algún } \\
\text { pariente suyo fue víctima de } \\
\text { la violencia delincuencial (Ej. } \\
\text { lesión u homicidio)? }\end{array}$ & $409(36,0)$ & $174(42.5)$ & $235(57.5)$ & $5.316^{*}$ \\
\hline $\begin{array}{l}4 \text { ¿Usted ha pensado en cambiar } \\
\text { su lugar de residencia, como } \\
\text { producto de la violencia } \\
\text { delincuencial? }\end{array}$ & 427(37.9) & $191(44.7)$ & $236(55.3)$ & 1.812 \\
\hline $\begin{array}{l}5 \text { ¿Algún familiar, amigo o } \\
\text { conocido ha cambiado su } \\
\text { lugar de residencia, como } \\
\text { resultado de la violencia? }\end{array}$ & $574(50.9)$ & $274(47.9)$ & $299(52.1)$ & 0.233 \\
\hline $\begin{array}{l}6 \text { ¿En su casa, se han } \\
\text { implantado algunas medidas } \\
\text { de seguridad? (Ej.: cerrar } \\
\text { temprano la puerta, no estar } \\
\text { en la calle.) }\end{array}$ & $859(76.4)$ & $39946.4)$ & $46053.6)$ & 0.639 \\
\hline $\begin{array}{c}7 \text { ¿Considera usted que las } \\
\text { noticias que transmiten por } \\
\text { televisión, prensa escrita } \\
\text { y radio sobre la violencia } \\
\text { delincuencial (Ej.: lesionados, } \\
\text { muertos, extorsiones) afectan } \\
\text { su estado emocional y mental? }\end{array}$ & $868(76.5)$ & $387(44.6)$ & $481(55.4)$ & $9.317^{* *}$ \\
\hline
\end{tabular}




\begin{tabular}{|c|c|c|c|c|c|}
\hline \multirow{2}{*}{\multicolumn{2}{|c|}{ Ítems }} & \multicolumn{4}{|c|}{$\begin{array}{l}\text { Frecuentemente } \\
\text { Siempre }\end{array}$} \\
\hline & & $\begin{array}{l}\text { Total } \\
(\%)\end{array}$ & $\begin{array}{c}\text { Hombres } \\
(\%)\end{array}$ & $\begin{array}{l}\text { Mujeres } \\
(\%)\end{array}$ & $X^{2}$ \\
\hline & $\begin{array}{l}\text { ¿En el último año, ha } \\
\text { modificado su vida cotidiana } \\
\text { debido a la violencia } \\
\text { delincuencial? (Ej.: llegar } \\
\text { temprano a casa, no salir de } \\
\text { noche.) }\end{array}$ & $855(75.6)$ & $397(46.4)$ & $458(53.6)$ & 0.376 \\
\hline & $\begin{array}{l}\text { ¿Siente temor de transitar } \\
\text { por algunas calles o zonas de } \\
\text { la ciudad (colonias, barrios o } \\
\text { pueblo)? }\end{array}$ & $922(81.3)$ & $415(45.0)$ & $507(55.0)$ & $7.848^{* *}$ \\
\hline & $\begin{array}{l}\text { ¿El contexto de violencia } \\
\text { delincuencial que se vive } \\
\text { en El Salvador le produce } \\
\text { ansiedad y estrés (pánico, } \\
\text { miedo, agitación, boca seca, } \\
\text { inquietud, taquicardia)? }\end{array}$ & $866(76.4)$ & $392(45.3)$ & $474(54.7)$ & $5.135^{*}$ \\
\hline & $\begin{array}{l}\text { ¿El ambiente de violencia } \\
\text { delincuencial que se vive } \\
\text { en El Salvador le produce } \\
\text { nerviosismo e inseguridad? }\end{array}$ & $961(84.8)$ & $436(45.4)$ & $525(54.6)$ & $7.119^{* *}$ \\
\hline & $\begin{array}{l}2 \text { ¿Considera usted que la } \\
\text { situación de violencia } \\
\text { delincuencial que vive el país } \\
\text { le afecta el sueño? }\end{array}$ & $751(65.9)$ & $326(43.4)$ & $425(56.6)$ & $11.506^{* *}$ \\
\hline & $\begin{array}{l}3 \text { ¿El contexto de violencia } \\
\text { delincuencial que se vive en El } \\
\text { Salvador afecta su estabilidad } \\
\text { emocional y mental? }\end{array}$ & $807(70.7)$ & $347(43.0)$ & $460(57.0)$ & $17.511^{* * *}$ \\
\hline
\end{tabular}

Nota: ${ }^{*} p<.05 ;{ }^{* *} p<.01 ;{ }^{* * *} p<.001$

Asimismo, el estudio señala que el $26.6 \%$ de la muestra informó que fue víctima directa de la violencia delincuencial en el último año. Este grupo de participantes reportó que los delitos más frecuentes que sufrieron fueron: robo (56.7\%), lesión e homicidio (36\%) y 
extorsiones (19\%). Con relación al lugar en donde ocurrió el delito, el $51.6 \%$ expreso que fue en la calle de las ciudades, mientras que el $5.7 \%$ manifestó que los delitos sucedieron en los pueblos.

En referencia al contexto de violencia delincuencial en el que vive la población, $797(70.6 \%)$ personas manifestaron que frecuentemente afecta su salud mental, mientras que el 866 (76.4\%) de la muestra consideró que el contexto de violencia social delincuencial le producía ansiedad y estrés (pánico, miedo, agitación, boca seca, inquietud, taquicardia). A su vez, se encontraron asociaciones significativas entre el sexo y ambas alteraciones mentales, presentando mayores dificultades de salud mental las mujeres en comparación con los hombres. Al preguntar a los participantes si sentían temor de transitar por algunas calles o zonas de la ciudad (colonias, barrios o pueblo), 922 (81.3\%) expresaron que frecuentemente $(45.0 \%$ hombres y $55.0 \%$ mujeres).

Con relación a la pregunta respecto a si la valoración de las noticias que se transmiten por televisión, prensa escrita y radio sobre la violencia social delincuencial (lesionados, muertos, extorsiones), afectaba su estado emocional y mental, 868(76.5\%) manifestaron que frecuentemente.

$\mathrm{Al}$ analizar el estrés y la ansiedad delincuencial en función al sexo, se encontró que existe una mayor incidencia en las mujeres. Al contrastar estas dimensiones en función del lugar de residencia, se observa que existe una mayor prevalencia en la población urbana en comparación con la población rural. Lo anterior indica claramente los efectos de la violencia social delincuencial en los problemas de salud mental y bienestar psicológico en la población, presentándose una mayor incidencia en los residentes de la ciudad que en los residentes rurales.

Con respecto a los indicadores de problemas de salud mental y bienestar psicológico que con frecuencia presenta la muestra del estudio, se evalúo si las preocupaciones les hacían perder mucho sueño a los participantes, encontrando que 301 (26.4\%) expresaron que les afectaba frecuentemente; de estos, 142 (41.2\%) fueron hombres y 177 (58.8\%) fueron mujeres. En el caso del ítem sobre si se han sentido agobiados y con tensión en los últimos días, 345 (30.5\%) manifestaron 
que con frecuencia ( $41.4 \%$ hombres y $58.6 \%$ son mujeres). Asimismo, se les preguntó si habían sentido que no podían superar las dificultades personales, encontrando que $347(30.6 \%)$ consideraban que sentían esto frecuentemente, de los cuales 160 (46.1\%) fueron hombres y 187 (53.9\%) fueron mujeres. Con respecto a si se han sentido poco felices y deprimidos, $244(21.5 \%)$ expresaron que frecuentemente $(42.6 \%$ hombres y $57.4 \%$ son mujeres). En todos los indicadores de problemas de salud mental comparados por sexo, se observan porcentajes más altos en las mujeres, mostrando que existen más dificultades de salud mental en este grupo.

De acuerdo con la prueba de correlaciones de Pearson ( $r$ ), se hallaron correlaciones positivas significativas entre las variables estrés delincuencial y salud mental $(r=.317 ; p=.01)$, ansiedad delincuencial y salud mental $(r=.427 ; p=.01)$, y entre sexo y ansiedad delincuencial $(r=.105 ; p=.01)$. A su vez, se encontraron correlaciones negativas significativas entre las variables ser víctima de violencia delincuencial y estrés delincuencial $(r=-.363 ; p=.01)$, y entre ser víctima de violencia delincuencial y la ansiedad delincuencial $(r=-.205 ; p=.01)$. La Tabla A1 ilustra estos datos, indicando que tanto el sexo de los participantes, el estado familiar, el nivel educativo, el hecho de ser víctima de violencia delincuencial, el área de trabajo, el maltrato familiar y la violencia estructural, están asociadas con la violencia social delincuencial.

$\mathrm{El}$ análisis inferencial realizado mediante la prueba $t$ de Student para muestras independientes en estrés delincuencial, ansiedad delincuencial y salud mental, en función del sexo de los participantes, reveló que al comparar la ansiedad delincuencial se encontraban diferencias significativas entre hombres y mujeres $(M=16.75, D E=5.60 ; M=$ 17.91, $D E=5.41), t_{(1,141)}=-3.555, p<.001$, indicando de que existen mayores niveles de ansiedad delincuencial en las mujeres. No se encontraron diferencias significativas en estrés delincuencial en función del sexo $(M=11.41, D E=4.19), t_{(1,141)}=-.695, p=.487$. Al contrastar la salud mental en función del sexo de la muestra, se encontró de que hay diferencias estadísticas significativas entre hombres y mujeres $(M=$ $11.45, D E=3.36 ; M=12.41, D E=3.51), t_{(1,141)}=-4.627, p<.001$, 
siendo las mujeres quienes presentan un mayor número de síntomas de problemas de salud mental.

Al contrastar mediante la prueba $t$ de Student para muestras independientes las dimensiones de estrés delincuencial, ansiedad delincuencial y salud mental, en función de si el participante fue víctima de violencia delincuencial en el último año, se encontró que había diferencias significativas en estrés delincuencial entre las víctimas y las no víctimas de la delincuencia $(M=14.09, D E=4.88 ; M=10.59$, $D E=3.66), t_{(1,131)}=11,110, p<.001$. A su vez, se encontraron diferencias significativas en ansiedad delincuencial al comparar las víctimas y las no víctimas $(M=19.24, D E=5.27 ; M=16.68, D E=5.48)$, $t_{(1,131)}=7,057, p<.001$, indicando que presentan mayores niveles de estrés y ansiedad delincuencial las personas que fueron víctimas de la violencia. Por otra parte, al contrastar la salud mental en función de si fue víctima o no de la violencia delincuencial, la prueba $t$ para muestras independientes reveló que existen diferencias significativas entre estos dos grupos $(M=12.72, D E=3.80 ; M=11.69, D E=3.30)$, $t_{(1,131)}=4,453, p<.001$; presentando mayor número de síntomas de problemas de salud mental aquellas personas que fueron víctimas de la violencia delincuencial.

$\mathrm{El}$ análisis de regresión logística proporcionó un modelo significativo, $F_{(4,112)} 191.41, p<.001$, para el que $R=.64$ y el coeficiente de determinación corregido $R^{2}=.41$. El modelo de regresión múltiple por pasos sucesivos resultó en cuatro modelos significativos, que se pueden observar en la Tabla 2. 


\section{Tabla 2}

Resumen del modelo de regresión obtenido para explicar la ansiedad delincuencial, como indicador sintomático de salud mental en la población salvadoreña víctima de la violencia social delincuencial

\begin{tabular}{|c|c|c|c|c|c|c|c|c|c|}
\hline Modelo & $R$ & $\begin{array}{c}R^{2} \\
\text { corregida }\end{array}$ & $B$ & Beta & $T$ & $p$ & $\begin{array}{c}r \\
\text { parcial }\end{array}$ & $\begin{array}{l}r \text { semi- } \\
\text { parcial }\end{array}$ & Tolerancia \\
\hline 1 & .51 & .26 & .46 & .36 & 19.87 & .001 & .39 & .33 & 1.00 \\
\hline 2 & .58 & .34 & .37 & .27 & 11.59 & .001 & .32 & .26 & .93 \\
\hline 3 & .64 & .40 & .41 & .26 & 10.86 & .001 & .30 & .24 & .89 \\
\hline 4 & .64 & .41 & .69 & .06 & 2.67 & .008 & .08 & .06 & .98 \\
\hline
\end{tabular}

En el modelo 1, la variable introducida fue estrés delincuencial, la cual explicó el 26\% de la ansiedad delincuencial y un coeficiente de correlación semiparcial de $r=.33$. En el segundo modelo se ańade la variable violencia sociocultural (estructural), sumando una varianza de explicación de $8 \%$ en la ansiedad delincuencial, con un coeficiente de correlación semiparcial de $r=.26$. En el tercer modelo se añadió la variable deterioro de la salud mental, agregando una varianza explicada del 6\% en la expresión de ansiedad delincuencial, con un coeficiente de correlación semiparcial de $r=.24$. Para el cuarto modelo se ańade la variable sexo, que eleva la varianza total explicada a un total de $41 \%$ de ansiedad delincuencial, con un coeficiente de correlación semiparcial de $r=.06$. Para un nivel del 95\%, el modelo que incluye las cuatros variables independientes fue significativo (ver figura 1).

Los coeficientes Beta reflejan el impacto relativo de las variables sobre la ansiedad delincuencial. En orden de influencia sobre la variable criterio, se situaron: estrés delincuencial $(B=.36)$, violencia sociocultural $(B=.27)$, deterioro de la salud mental $(B=.26)$, y sexo $(B=.06)$. El orden se mantuvo en la correlación parcial $(r=.39, r=.32, r=.30$, $r=.08)$, y semiparcial $(r=.33, r=.26, \mathrm{r}=.24, r=.06)$. 


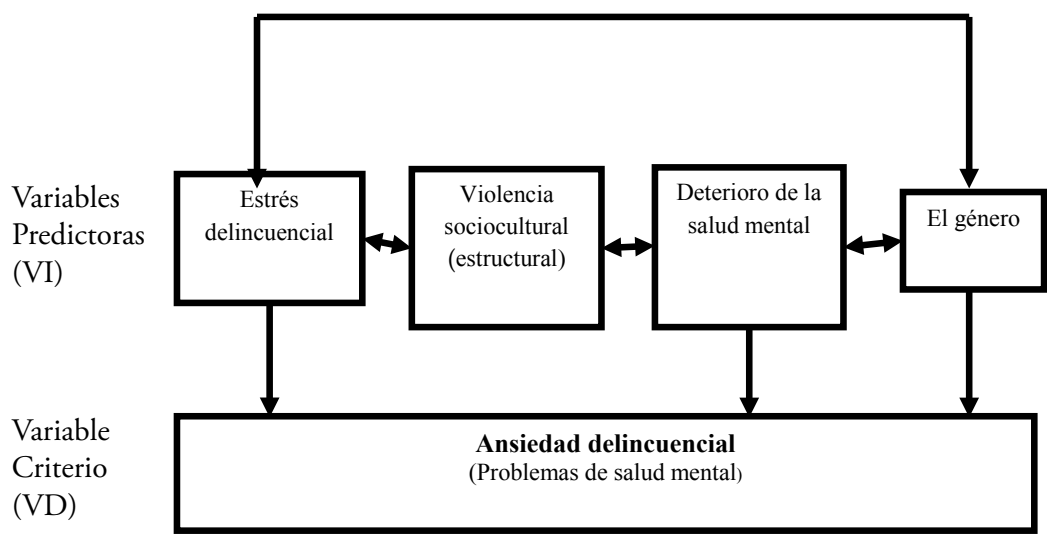

Figura 1. Flujograma que explica la ansiedad delincuencial en la población salvadoreña víctima de la violencia social delincuencial

\section{Discusión}

Un resultado importante de este estudio es que el contexto de violencia social delincuencial es un factor psicosocial que frecuentemente afecta la estabilidad psíquica, produciendo alteraciones como: nerviosismos, inseguridad, inestabilidad emocional y falta de salud mental en la población salvadoreña; presentándose una mayor incidencia las mujeres en comparación con los hombres. Cerca de tres cuartas partes de la muestra presentan altos niveles de estrés y ansiedad delincuencial. Es probable que gran parte las víctimas antes señaladas sean, su vez, víctimas indirectas de la violencia social delincuencial.

Del mismo modo, más de una cuarta parte de la muestra informó haber sido víctima directa de la violencia delincuencial en el último año. Estos resultados sugieren que la violencia delincuencial afecta la estabilidad psicológica, tanto de las víctimas directas como de las víctimas indirectas de la violencia social delincuencial, afectando mayoritariamente a quienes viven en las ciudades en contraste con los que viven en los pueblos y áreas rurales. De esta manera, el mayor número de delitos es cometido en las calles de las ciudades y las residencias (casas), 
mientras que los lugares de menor riesgo delincuencial son los pueblos. Briseño (2007) menciona que la situación de violencia ha llevado a un sentimiento de inseguridad muy alto en las distintas zonas de la ciudad, produciendo que los estilos de vida de los habitantes se vean alterados con el fin de adaptarse a las condiciones de inseguridad que perciben.

Con relación a la frecuencia de los tipos delitos en las víctimas directas, estos suceden en el siguiente orden: robos, lesión-homicidios, extorsiones, hurtos y asaltos. Lo encontrado es coherente con lo hallado por el Centro de Investigación de la Opinión Pública Salvadoreña (CIOPS, 2011), en donde también se concluyó que los lugares en los que se cometían mayormente los delitos eran en las calles y en las residencias.

Por otra parte, el estudio revela que una inmensa mayoría de la muestra de salvadoreńos expresó que las noticias que se transmiten por los diversos medios de comunicación social (televisión, prensa escrita y la radio) sobre la violencia social delincuencial (lesionados, muertos, robos y extorsiones) afectan su estado emocional y mental, indicando que la exposición constante a hechos violentos transmitidos por los medios de comunicación pueden estar incrementando los problemas de salud mental de la población. Estos resultados están en sintonía con lo expuesto por Huhn y colaboradores (2007), quienes plantean que la violencia en sí misma no es la que tienen más influencia en las opiniones y en la sensación de inseguridad en una sociedad, sino más bien los discursos y prácticas sociales. Por lo tanto, los mensajes transmitidos por los medios de comunicación social sobre la violencia social delincuencial pueden estar provocando temor en la población, a pesar de que el número de homicidios haya disminuido.

Los datos revelan alta prevalencia de estrés y ansiedad delincuencial en la muestra, encontrando una mayor incidencia en las mujeres. Por otra parte, es importante subrayar que existe una prevalencia más alta de estrés y ansiedad delincuencial en la población que reside en las ciudades en comparación con las personas que residen en las zonas rural. Probablemente lo anterior se deba a que la mayoría de los delitos ocurren en las calles y las residencias de las ciudades. Además, esto podría ser atribuido a que las "pandillas" se ubican principalmente en 
las áreas urbanas y suburbana (Cabezas et al., 2008; Falkenburger \& Thale, 2008). Sin embargo, cabe destacar que el contexto de violencia social delincuencial afecta significativamente a la población general, pero de forma más pronunciada a los residentes de las ciudades y al género femenino. Como consecuencia de lo antes señalado, la violencia social delincuencial conformaría un factor psicosocial que afecta significativamente la salud mental y el bienestar psicológico de la población salvadoreña.

Estos hallazgos concuerdan con lo encontrado por Lederbogen y colaboradores (2011), quienes consideran que las personas que viven en la ciudad, a diferencia de los que viven en zonas rurales, tienen más probabilidades de sufrir más estrés y enfermedades mentales. A su vez, Kessler y colaboradores (2003) señalan que entre los factores asociados a la ansiedad está el ser mujer.

Entorno a lo expuesto, el estudio revela que cerca de una tercera parte de la muestra presenta diferentes síntomas de problemas de salud mental, existiendo una mayor prevalencia e indicadores de problemas de salud mental en las mujeres y en los residentes urbanos. Entre los indicadores de falta de salud mental se encuentran los niveles de ansiedad y estrés delincuencial antes señalados. Estos hallazgos están en relación con lo encontrado en un estudio realizado a escala nacional (Gutiérrez, 2011) que halló que la ansiedad y la depresión son las dos alteraciones mentales más prevalentes en la población salvadoreńa. En este trabajo, más de la mitad de la muestra presentó síntomas de ansiedad e insomnio, y un mayor número de síntomas lo presentaron las mujeres y los habitantes de las áreas rurales. Igualmente, entre los años 2006-2012, la ansiedad y la depresión aparecen en primer y segundo lugar respectivamente como las principales causas de consulta de los trastornos mentales y del comportamiento (Minsal, 2012).

Los datos demuestran que el estrés y la ansiedad delincuencial están asociados con la salud mental de la población salvadoreña, encontrándose una correlación significativa moderada entre el estrés delincuencial y la ansiedad delincuencial con la salud mental. A su vez, existe una correlación significativa entre las anteriores variables 
con el género, el estado familiar, el nivel educativo, haber sido víctima de violencia delincuencial, el área de trabajo, el maltrato familiar y la violencia sociocultural. Estos hallazgos son apoyados por el estudio de Gutiérrez (2011), quien encontró una correlación significativa entre las variables sociodemográficas con la salud mental en una muestra salvadoreña. Como consecuencia es relevante considerar lo planteado por Slavich y Taylor (2010) quienes expresan que la exposición a grandes cantidades de estrés podría cambiar la forma en la que el sistema inmune responde a las amenazas exteriores. Estos cambios pueden abrir el camino a muchas enfermedades o infecciones, y podrían desencadenar una variedad de alteraciones mentales como: ansiedad generalizada (fobias), insomnio, depresión, alteraciones psicosomáticas y, en algunos casos, trastornos paranoide de la personalidad y estrés pos traumático. Por ello, disminuir el estrés y la ansiedad delincuencial debería ser una prioridad en el país.

Es importante mencionar, que una concentración excesiva de cortisol puede perjudicar estructuras cerebrales y determinados neurotransmisores, alterando el funcionamiento cerebral y el comportamiento humano. Los hallazgos de Johnson (2002) señalan una correlación entre la ansiedad y los estilos de vida que afectan la salud, por ejemplo el consumo de alcohol, tabaco y drogas. Asimismo, la ansiedad se ha asociada al riesgo de desarrollar ciertas patologías, como la hipertensión en las mujeres (Adler \& Matthews, 1994) o el factor de riesgo de la sobre mortalidad cardiovascular (Hansen, 2003). Del mismo modo, si el sentimiento de carácter negativo aparece de forma constante en el sujeto y no es tratado adecuadamente, puede conducir a un bajo rendimiento en la vida cotidiana, debilitando la salud (Olga \& Terry, 1997). Hudd et al. (2000) encontraron que los estudiantes con niveles más altos de estrés mostraban peores hábitos de salud, autoestima más baja y mala percepción de su estado de salud. Lo anterior indica que los altos niveles de estrés y ansiedad afectan la salud física y mental de las personas, así como su calidad de vida.

El análisis inferencial mediante la comparación de medias demostró que existen diferencias significativas en ansiedad delincuencial y 
salud mental en función del género, siendo las mujeres quienes presentan una mayor incidencia de síntomas y de problemas de salud mental asociados a la violencia delincuencial. Similares resultados se encontraron al comparar estas variables de bienestar psicológico (salud mental) en función del lugar de residencia, presentando una mayor prevalencia de problemas de salud mental relacionados con la violencia delincuencial los residentes urbanos.

Al mismo tiempo, se encontraron mayores problemas de salud mental, mayores niveles de estrés y ansiedad delincuencial en quienes fueron víctimas de la violencia delincuencial en comparación con las personas que no lo fueron. En general, los hallazgos sugieren que un contexto de violencia social delincuencial, donde existe una exposición frecuente a eventos emocionalmente traumáticos tales como: ser víctima de amenazas, robos, hurtos, extorsiones, lesiones y asesinatos, es un factor de alto riesgo que puede desencadenar una serie de afecciones de naturaleza psíquica en las víctimas directas o indirectas de la violencia delincuencial. Asimismo, se aprecia que este contexto de violencia está modificando los estilos de vidas de las familias salvadoreñas, lo que se evidencia en el abandono de muchas zonas (colonias, barrios) por temor a ser víctimas de la delincuencia, las restricciones de acceso a colonias, condominios vigilados, el pago de vigilancia privada y el no frecuentar lugares identificados como peligrosos en la calles o ciudad (Huhn et al., 2007).

Un porcentaje importante de la ansiedad delincuencial que experimenta la población salvadoreńa se explica mediante un análisis de regresión (modelo de regresión logística) que resultó en cuatro modelos significativos, donde las variables predictoras fueron: estrés delincuencial, violencia estructural, deterioro de la salud mental y el sexo. En conjunto las cuatro variables explican un $41 \%$ de la ansiedad delincuencial.

Es oportuno destacar que el propósito de analizar la ansiedad delincuencial como dimensión o factor empírico de la afectación de la salud mental en la población víctima directa o indirecta de la violencia social delincuencial, fue cuantificar los efectos de la violencia delincuencial en la salud mental de la población salvadoreña. Estos resultados llevan a 
pronosticar que aquellas personas que experimenten condiciones similares de violencia social delincuencial podrían presentar alteraciones importantes en su salud mental. Asimismo, un sistema de salud con pobre calidad de atención, un sistema de justicia incapaz de controlar y prevenir el delito, una población con problemas económicos y la existencia de conflictos políticos, contribuyen al incremento de la violencia delincuencial y de los problemas de salud (mental) en la población. De esta manera, la prevención de la violencia y de los problemas de salud general, no sólo benefician a los individuos, sino también a sus familias, a la comunidad y a la economía del país. También generan mejoras en la educación, el trabajo y contribuyen al desarrollo humano y social, siendo la salud mental la base para el bienestar y funcionamiento efectivo de las personas y la sociedad (Moll, 2013).

En síntesis se puede decir que un contexto de violencia social delincuencial es un factor desencadenante de alteraciones emocionales y psicológicas en la población, y que los mensajes emitidos por los medios de comunicación sobre dicho factor inciden en la salud mental de la población.

\section{Referencias}

Adler, N. \& Matthews, K. (1994). Health Psychology: why do some people get sick and some stay well? AnnualReview of Psychology, 45(1), 229-259.

Banco Mundial, Departamentos de Desarrollo Sostenible y Reducción de la Pobreza y Gestión Económica; Región de América Latina y el Caribe. (2011). Crimen y violencia en Centroamérica. Un desafío para el Desarrollo.

Briceño, R. (2007). Violencia Urbana en América Latina: Un modelo sociológico de explicación. Espacio Abierto Cuaderno Venezolano de Sociología, 16(3), 541- 574.

Cabezas, R., Gilsanz, J. \& Sampayo, L. (2008). Cohesión social y educación para la paz: Alternativas de prevención de la 
violencia infanto-juvenil en El Salvador. Revista CIDOB d'Afers Internacionals, 81, 91-108.

Calzada, A. (2007). Aproximación a los correlatos biológicos de la agresividad y la violencia humana. Revista Neurología, Neurocirugia y Psiquiatría, 40(4), 114-121.

Centro de Investigación de la Opinión Pública Salvadoreña (CIOPS). (2011). Encuesta de opinión sobre aspectos económicos, politicos y sociales del pais. Universidad Tecnológica de El Salvador, San Salvador.

Centro de Estudios sobre Estrés Humano (CSHS). (2010). Comprender el estrés. Recuperado de:http://translate.google.com/ translate?depth=1\&hl=es\&prev=/search $\% 3 \mathrm{Fq} \% 3 \mathrm{DS}$ onia $\% 2 \mathrm{BL}$ upien $\% 2$ Bstress $\% 26$ client $\% 3$ Dfirefoxa $\% 26 \mathrm{hs} \% 3 \mathrm{DAVX} \% 26 \mathrm{~s}$ a\%3DX\%26rls\%3Dorg.mozilla:esES:official\%26channel\%3D np\%26biw\%3D1366\%26bih\%3D638\&rurl=translate.google. com.sv\&sl=en\&u=http://www.humanstress.ca/stress/what-isstress/biology-of-stress.html

Cervantes, R.C., Salgado de Zinder, V.N. \& Padilla, V.M. (1989). Posttraumatic Stress in Immigrants for Central America and México. Hops Community Psychitry, 40 (6), 615-619.

Echeburúa, E., Corral, P., Amor, P., Sarasua, B. \& Zubizarreta, I. (1997). Repercusiones psicopatológicas de la violencia doméstica en la mujer: un estudio descriptivo. Revista de Psicopatología y Psicología Clínica, 2(1), 7-19.

Falkenburger, E. \& Thale, G. (2008). Maras centroamericanas: Politicas públicas y mejores prácticas. Revista CIDOB d'Afers Internacionals, 81,45-66.

Gutiérrez, J.R. (2011). Prevalencia de alteraciones mentales: depresión y ansiedad, en la población salvadoreña. Estado de la salud mental. Universidad Tecnológica de El Salvador: Tecno impresos, San Salvador.

Gutiérrez-Quintanilla, J.R. (2012). La violencia social delincuencial relacionada con la salud mental en los salvadoreños editorial. San Salvador: Tecnoimpresos. 
Hansen, S. (2003). Mental health issues associated with cardiovascular disease in women. Psychiatric clinics of North America, 26(2), 693-712.

Hudd, S., Dumlao, J., Erdmann-Sager, D., Murray, D., Phan, E., Soukas, N. \& Yokozuka, N. (2000). Stress at college: effects on health habits, health status and self-esteem. Canadian Journal of Occupational Therapy, 67(4), 230-238.

Huhn, S., Oettler, A. \& Peetz, P. (2007). La construcción de realidades inseguras. Reflexiones acerca de la violencia en Centroamérica. Revista de Ciencias Sociales, 117, 73-89.

Instituto Universitario de Opinión Pública (IUDOP). (2007). Los salvadoreños y salvadoreñas evalúan la situación del país a finales de 2007. Boletín de Prensa. Año XXII, n. ${ }^{\circ 2}$. San Salvador: Universidad Centroamericana "José Simeón Cañas."

Johnson, R.L. (2002). The relationships among racial identity, selfesteem, sociodemographics and health-promoting lifestyles. Research and Theory for Nursing Practice, 16 (3), 193-207.

Kessler, R., Berglund, P., Demler, O., Jin, R., Koretz, D., Merikangas, K., Bush, J., Walters, E. \& Wang, P. (2003). The epidemiology of major depressive disorder. Results from the National Comorbity Survey Replication (NCS-R). JAMA, 289(23), 3095-105.

La Declaración de Ginebra sobre Violencia Armada y Desarrollo (2011). Carga Global de la Violencia Armada 2011 Encuentros Letales. Recuperado de: http://www.genevadeclaration.org/ fileadmin/docs/GBAV2/GBAV2011-Ex-summary-SPA.pdf

Lazarus, R. S. \& Folkman, S. (1986). Estrés y procesos cognitivos. Barcelona: Martínez Roca.

Lederbogen, F., Kirsch, P., Haddad, L., Streit, F.,_Tost, H., Schuch, F., Wüst, S.,_Pruessner, J., Rietschel, M., Deuschle, M. \& MeyerLindenberg, A. (2011). City living and urban upbringing affect neural social stress processing in humans. Nature International Weekly Journal of Science. 474, 498-501. doi: 10.1038/ nature 10190

Miguel-Tobal, J. J. (1996). La ansiedad. Madrid: Santillana. 
Ministerio de Salud (Minsal). (2003). Estadisticas nacionales de atención primaria en salud. San Salvador: El Salvador.

Ministerio de Salud (Minsal). (2010). Estadísticas nacionales de atención primaria en salud, Suicidios e intentos de suicidio. San Salvador: El Salvador.

Ministerio de Salud Pública (Minsal). (2012). Casos de trastornos mentales y del comportamiento notificados en los reportes de vigilancia semanal, El Salvador 2005-2012. San Salvador: El Salvador.

Moll, S. (2013). Representaciones de la salud mental en trabajadores de dos instituciones especializadas de Lima Metropolitana. Revista de Psicología, 31(1), 99-128.

Montero, I. \& León, O.G. (2007). A guide for naming research studies in Psychology. International Journal of Clinical and Health Psychology, 7, 847-862.

Morales, L., Muñoz, J., Santillán, A., Arenas, R. \& Ponce, F. (2007). Perfiles criminológicos: el arte de Sherlock Holmes en el siglo XXI. Salud Mental, 30(3), 68-75.

Mora, E., Borges, G., Lara, C., Ramos, L., Zambrano, J. \& Fleiz, C. (2005). Prevalencia de sucesos violentos y de trastorno por estrés postraumático en la población mexicana. Salud pública de México, 47(1), 8-22.

Morelato, G. (2011). Resiliencia en el maltrato infantil: aportes para la comprensión de factores desde un modelo ecológico. Revista de Psicología, 29 (2), 203-224.

Olga, G. \& Terry, L. (1997). Superar el estrés. Madrid: Ediciones Pirámide.

Organización Mundial para la Salud (2002). Informe mundial sobre la violencia y salud: resumen. Washington, D.C.: OPS. Recuperado de: http://www.who.int/violence_injury_prevention/ violence/world_report/es/summary_es.pdf

Organización Mundial de la Salud (OMS). (2003). Informe mundial sobre la violencia y la salud. Washington, DC: Autor. 
Organización Mundial de la Salud (OMS). (2011). Salud mental: un estado de bienestar. Recuperado de: http://www.who.int/features/ factfiles/mental_health/es/index.html

Policía Nacional Civil de El Salvador. (2011). Estadísticas de delitos cometidos en El Salvador, San Salvador.

Posada, R. \& Parales, C.J. (2012). Violencia y desarrollo social: más allá de una perspectiva de trauma. Universitas Psychologica, 11(1), 255-267.

Punset, E., (10 de noviembre de 2004). Entrevista de Robert Sapolsky. Estrés y Placer, extremos encontrados. Blog de Eduardo Punset, disponible en: http://www.eduardpunset.es/413/charlas-con/ estrs-y-placer-extremos-encontrados

Punset, E. (2006) El alma está en el cerebro. Madrid: Aguilar

Reyes, L. (2007). Enfermedad mental y violencia. Propuestas de Intervención en Enfermería. Rev Cubana Enfermer, 23(4), 14-8.

Saavedra, A. (2004). Violencia y salud mental. Acta Médica Peruana, 21(1), 39-50.

Sue, D. (1996). Comportamiento anormal. México, D.F: McGraw-Hill. Slavich, G. \& Taylor, C. (2010). El estrés social afecta el sistema inmune, Center for Psychoneuroimmunology de la Universidad de California. (UCLA).

United States Agency for International Development (USAID). (2006). Central America and Mexico Gang Assessment. USAID Bureau for Latin American and Caribbean Affairs, Office of Regional Sustainable Development.

Recibido: 13 de octubre, 2013 Aceptado: 20 de noviembre, 2013 


\section{Anexos}

\section{Tabla A1}

Correlaciones de estrés delincuencial, ansiedad delincuencial, con la salud mental y otras variables relacionadas.

\begin{tabular}{|c|c|c|}
\hline Variables/Dimensiones & $\begin{array}{c}\text { Estrés } \\
\text { delincuencial } \\
r\end{array}$ & $\begin{array}{c}\text { Ansiedad } \\
\text { delincuencial } \\
r\end{array}$ \\
\hline Sexo & .02 & $.11^{* *}$ \\
\hline Estado civil (familiar) & $.10^{* *}$ & $.11^{* *}$ \\
\hline Escolaridad & $.12^{* *}$ & -.04 \\
\hline Edad & .05 & $.06^{*}$ \\
\hline Sector de trabajo & $.13^{* *}$ & $.06^{*}$ \\
\hline Trabaja (Sí, No) & $-.08^{* *}$ & -.02 \\
\hline Víctima de violencia delincuencial & $-.36^{* *}$ & $-.21^{* *}$ \\
\hline Bienestar psicológico (Salud mental) & $.32^{* *}$ & $.43^{* *}$ \\
\hline Funcionamiento social & -.03 & -.02 \\
\hline Funcionamiento familiar & -.04 & .01 \\
\hline Abuso o maltrato familiar & $-.25^{* *}$ & $-.15^{* *}$ \\
\hline Violencia estructural (delincuencial) & $.26^{* *}$ & $.40^{* *}$ \\
\hline Consumo de droga/alcohol & .03 & .01 \\
\hline
\end{tabular}

Nota: ${ }^{*} p<.05 ;{ }^{* *} p<.01$ 